\title{
Numerical modeling of a high-enthalpy shock tunnel driven by gaseous detonation
}

\author{
Kai Luo ${ }^{\mathrm{a}, \mathrm{b}}$, Qiu Wang ${ }^{\mathrm{a}, *}$, Jiwei $\mathrm{Li}^{\mathrm{a}}$, Jinping $\mathrm{Li}^{\mathrm{a}, *}$, Wei Zhao ${ }^{\mathrm{a}, \mathrm{b}}$ \\ a State Key Laboratory of High Temperature Gas Dynamics, Institute of Mechanics, Chinese Academy of Sciences, No. 15 Beisihuanxi Road, Beijing, 100190, China \\ b School of Engineering Science, University of Chinese Academy of Sciences, No. 19 Yuquan Road, Beijing, 100049, China
}

\section{A R T I C L E I N F O}

\section{Article history:}

Received 28 November 2019

Received in revised form 2 April 2020

Accepted 2 April 2020

Available online 12 June 2020

Communicated by Kostas Kontis

\section{Keywords:}

Quasi-one-dimensional

Detonation

Shock tunnel

Shock speed

\begin{abstract}
A B S T R A C T
A detonation-driven shock tunnel is useful as a ground test facility for hypersonic flow research. Numerical modeling of the shock tunnel is an easy way to extract precise test flow conditions and generate predictions of new operating conditions. In this study, a quasi-one-dimensional modeling of a detonation-driven shock tunnel is described. The model accounts for the viscous effects within a shock tube by adding a source term. The simulations of the particular conditions of the detonation shock tunnel are then presented and compared with the experimental results. The simulations provide good estimates for both the shock speed and pressure distributions. Detailed information on the gas dynamic processes over the full length of the facility is also obtained and discussed. The results also show that the approach is valid and ideal for the development of new tunnel operating conditions. It is also an ancillary tool for characterization of the reservoir parameters in a shock tube/tunnel, especially for the total temperature which are difficult to measure in such facilities.
\end{abstract}

(C) 2020 Elsevier Masson SAS. All rights reserved.

\section{Introduction}

High-enthalpy/high-velocity flows are a field of increasing research interest. This is mainly due to the very complex chemical and physical interactions that can involve excitation of internal degrees of freedom, chemical reactions, radiation, entropy layers (such as those occurring behind curved shocks), and viscous effects (such as transition, turbulence and flow separation). These phenomena and their interactions can have of first order influence on the aerothermodynamics of high-speed vehicles.

Besides computational efforts to understand the corresponding phenomena, shock tunnels have advantages for simulating these kinds of flows [1-4]. In view of enthalpy and pressure requirements for the shock tunnels to replicate the hypersonic flow conditions, they must incorporate a high-performance driver. Among the existing driving techniques, only a few meet the requirement of a high-performance driver. The detonation drivers, which are anticipated to become the optimal choice for current supersonic research considering the benefit-cost ratio, are capable of simultaneously producing high-enthalpy and high-pressure test flows in addition to simple operation $[5,6]$. The detonation driver was first proposed by Bird [7] and has since been investigated by many researchers [8-10]. With the success of several crucial techniques,

\footnotetext{
* Corresponding authors.

E-mail addresses: wangqiu@imech.ac.cn (Q. Wang), lijinping@imech.ac.cn (J. Li).
}

such as spontaneous strong ignition and attenuation of the reflected waves, it has been widely used in shock tubes and tunnels producing high-enthalpy flows for aerodynamic testing $[9,11]$. Such as in the JF12, currently the largest shock tunnel in the world, which is capable of reproducing the pure airflow with a test duration longer than $100 \mathrm{~ms}[12,13]$. Unfortunately, only a few studies focusing on detonation driven shock tunnels have been conducted using numerical simulations. With the remarkable advances in physical modeling and the computing power of computational fluid dynamics (CFD), there has been considerable interest in modeling of the shock tunnel driven by detonation, where the CFD can provide more detailed information on the test-flow conditions, besides being efficient and less costly. In addition, it is also a simple method for the identification and assessment of new operating parameters of the test conditions without risk to the facility and hardware, as well as for the validation of new design concepts. As it is well established, this is important for a detonation facility operating under the conditions of high temperature, high pressure and presence of flammable and explosive gases.

The performance of the shock tube would be affected by many factors, like the diaphragm opening effects, driver gas contaminations, shock/boundary layer interaction and tube wall roughness. A two or three-dimensional investigation of the shock tube flows by solving the unsteady Navier-Stokes equation coupled with the heat conduction equation is, of course, a more appropriate method. Several researchers have used axisymmetric, two-dimensional simulations of the unsteady Navier-Stokes equations to investigate the 
shock tube flows [14-17]. Although these methods give satisfactory results in certain aspects, the computer resources and simulation time required to account for all important phenomena are high even for the simple shock tube cases, not to mention the detonation tubes where complicated chemical reactions occur. Besides, this method is also very difficult to estimate the diaphragm opening effect or tube wall dissipation effects on the performance of the shock tube. Thus, a quasi-one-dimensional code is more attractive for engineering design due to their lower computational requirements.

Several researchers have also developed quasi-one-dimensional simulations to generate information about the transient gas dynamics in the short-duration high-speed flow test facilities, such as shock tubes and shock tunnels [18-21]. In such studies, the simulated pressures were compared with the experimental measurements, and good agreement was often reported. The well-known code is the quasi-one-dimensional Lagrangian L1d solver developed by P. Jacobs from the University of Queensland [18]. It can successfully describe the compression process within a free-piston shock tube driver and also in computing the trajectory of the shock wave within the shock tube [11-13]. However, the quasi-onedimensional simulations suffer from the drawback that it is not easy to simultaneously simulate both the correct shock speed in the shock tube and the equilibrium conditions after the shock reflection. Besides, unlike a free-piston shock tunnel, the detonationdriven shock tunnels do not have a piston; instead, they involve the detonation of the flammable mixtures in the detonation chamber and dissociation of the test gas (air in the present study) in the shock tube, which are rather complicated processes. Owens and Hanson investigated the attenuation of one-dimensional detonation waves and they payed special attention to the influence of heat transfer, friction and condensation on the performance of the detonation tube. However, the incident shock wave issues were not involved in their studies [22,23].

In the present study, a quasi-one-dimensional chemical equilibrium flow model was developed to simulate the flow process in a detonation shock tube. Viscous effects are incorporated by the well-known skin friction correlations for pipe flow. The reference temperature is used to correct the effects of the Mach number and the difference between the wall temperature. The heat transfer between the gas and wall is given by the modified Reynolds analogy for turbulent flow in pipes. Typical experiments are performed to validate the numerical method and good agreements are obtained. The unsteady gas dynamic processes over the full length of the facility are also discussed in detail.

\section{Numerical modeling}

\subsection{Facility specification}

This paper concerns the modeling and simulation of a particular facility, namely the JFX shock tunnel located in the State Key Laboratory of High Temperature Gas Dynamics (LHD), Institute of Mechanics, and Chinese Academy of Sciences (CAS). The facility consists of a dump section, a driver section, and a driven section, as shown in Fig. 1. The length of the driver and driven sections is 6.6 and $6.9 \mathrm{~m}$, respectively and both have an inner diameter of 126 $\mathrm{mm}$. The dump section, with an inner diameter of $345 \mathrm{~mm}$, is only used in the backward detonation driving mode to decrease the reflected shock pressure, which may damage the facility. The nozzle and test sections are not considered in the present study.

For the JFX shock tunnel, several common operation cases, shown in Table 1, are usually used by different modes of operation. Different experimental conditions represent the different capacities to replicate the JFX shock tunnel. The backward and forward

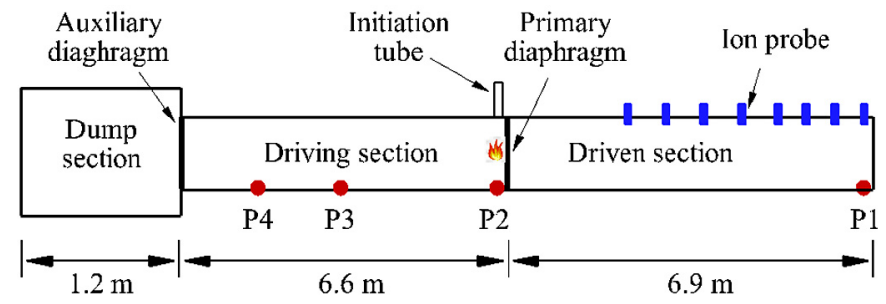

Fig. 1. Schematic diagram of the JFX detonation-driven shock tube.

Table 1

Typical operation state of JFX shock tunnel.

\begin{tabular}{|c|c|c|c|c|c|}
\hline Case & & 1 & 2 & 3 & 4 \\
\hline Driving mode & & \multicolumn{3}{|c|}{ Backward } & Forward \\
\hline Driving section & $\begin{array}{l}\text { Mixing ratio } \\
\mathrm{H}_{2}: \mathrm{O}_{2}: \mathrm{N}_{2}\end{array}$ & $2: 1: 1$ & $2: 1: 1$ & $4: 1$ & $2: 1: 1$ \\
\hline & Pressure (MPa) & 1.5 & 1.2 & 1.5 & 1.2 \\
\hline Driven section & Gas & \multicolumn{4}{|c|}{ Air } \\
\hline & Pressure (KPa) & 13 & 10 & 7 & 10 \\
\hline Total enthalpy (MJ/kg) & & 6.8 & 6.6 & 12 & 13.5 \\
\hline
\end{tabular}

detonation-driven systems basically depend on the different ignition positions in the driving section. The ignition position of the backward mode is located near the main diaphragm, while the forward detonation mode is initiated at the end of the driving section. Compared with the backward detonation mode, which can achieve stable test flows with a long driving time at a relatively low enthalpy level, the forward detonation mode can achieve the higher-enthalpy flows. According to the different hypersonic flow conditions that we want to replicate, we choose the appropriate operation mode to conduct the test. More detailed information on the JFX shock tunnel can be found in the literature [24]. In this paper, case 1 is selected as the quasi-one-dimensional numerical simulation model for analysis, and others are used for further verification. The reservoir conditions including the stagnation enthalpy is estimated from idealized zero-dimensional calculations of normal shock and isentropic processes, anchored to the measured shock speed and initial pressure/temperature values, while assuming thermochemical equilibrium for high enthalpy conditions. And it needs to be noted that the enthalpy in Table 1 is calculated with the incident shock speed at the centre part of the driven section.

For shock tunnels, the pressure and shock speed in the driver and driven section are the key parameters to evaluate the performance of the shock tunnels. Therefore, this paper focuses on the measurement of the incident shock wave velocity and pressure. Piezoelectric sensors were arranged in the driving section to monitor the pressure histories and the velocity of the detonation wave propagation. The incident shock speed is measured with several ion probes distributed along the driven section. At the same time, piezoresistance transducers are also mounted on the tube sidewall to record the pressure history. The distribution of the transducers is shown in Fig. 1. Stations P1, P2, P3 and P4 are the sensors that monitor the pressure histories, and other positions are mainly equipped with ion probes. In this one-dimensional formulation, the facility is modeled as a linear array of circular pipes aligned with the $x$ axis. The auxiliary diaphragm is located at $x=0$, and the primary diaphragm is located at $x=6.6 \mathrm{~m}$.

\subsection{Physical modeling}

\subsubsection{Governing equations}

The numerical method is based on a quasi-one-dimensional chemical equilibrium flow model, with the equations, written in conservation form, as follows: 
$A \frac{\partial U}{\partial t}+\frac{\partial A F(U)}{\partial x}-\frac{\partial A}{\partial x} H-S_{w}=0$

where $U=\left(\rho_{i}, \rho u, e\right)^{T}$ is the state vector, $F=\left(\rho_{i} u, \rho u^{2}+p\right.$, $(e+p) u)^{T}$ is the flux vector, $H=(0, p, 0)^{T}$ is the wall pressure source term, and $S_{w}=(0, \tau, q)$ is the wall-friction and heattransfer source term; where $\rho, u, e, p$, and $A$ are the density, velocity, total energy, pressure of gas, and cross-sectional area, respectively; $\tau$ is the wall-shear stress and $q$ is the wall-heat flux. The subscript " $i$ " denotes the species.

The spatial discretization is obtained using the finite difference method. The inviscid fluxes are computed using the dispersioncontrolled dissipation scheme proposed by Jiang [25]. The results of the simulations are also compared against the Euler case, with no shock deceleration.

\subsubsection{Equilibrium model}

During the operation of the detonation-driven high-enthalpy shock tunnel, a series of complex physical and chemical phenomena, such as detonation wave propagation, shock wave propagation, boundary layer interaction and non-equilibrium flow, are involved. In the numerical simulation, it is difficult to cover all aspects. Thus, the corresponding simplification can only be made according to the different aspects of the discussion. In this study, it is anticipated that the one-dimensional program can be used to accurately determine the internal wave motion process and relevant characteristic parameters of the shock tube without increasing the amount of calculation.

For hypersonic flows, the gas particle may begin to dissociate or ionize due to the high-temperature effect [26,27]. Under the hightemperature condition, the thermodynamic properties of the gas will differ far from those of the ideal gas. The effects of the dissociation and ionization of the gas on the thermodynamic properties are generally called real gas effects. In our shock tube cases, the temperature of gas in region 2 is not high enough to cause significant dissociation and ionization. In the region behind the reflected shock (region 5) where the dissociation and ionization can be considered significant, the flow velocity is almost static and the gas density is higher than that under the standard conditions, which means that the dissociation and recombination relaxation time will be even shorter [28]. Meanwhile, considering that the time scale associated with the finite-rate source terms in the non-equilibrium computations is approximately 500-10,000 times smaller than the gas dynamic time scale and the amount of calculation of the equilibrium model is twice less than that of the non-equilibrium model [29], the chemical reaction in our cases can be assumed to be in chemical equilibrium.

In all cases, the equilibrium composition of the gas also needs to be determined. The composition of equilibrium air in the present study is calculated using the minimization of the Gibbs free-energy method [30]. The thermodynamic properties of the individual species in the temperature range from 300 to $20,000 \mathrm{~K}$ were obtained from a report by the NASA Glenn Research Center [31], which provides thermodynamic data for over 2,000 chemical species. The thermodynamic properties provided are the $h, s$ and $\mathrm{cp}$, as a function of temperature, using polynomials with 9 coefficients. Once the thermodynamic properties of the individual species are stablished, the thermodynamic properties of the gas mixture can be determined using the ideal-gas-mixture rules, as discussed in the previous report [28].

The detonation process is accompanied by a strong chemical reaction, and its temperature is often higher than that of the incident shock wave [32,33]. In accordance with the air calculation method, the chemical equilibrium method is also used for the detonation gas. For oxyhydrogen detonation in the detonation chamber, seven components, $\mathrm{H}_{2}, \mathrm{O}_{2}, \mathrm{OH}, \mathrm{H}_{2} \mathrm{O}, \mathrm{HO}_{2}, \mathrm{O}$, and $\mathrm{H}$, are considered in the computations; for methane-oxygen detonation, $\mathrm{CH}_{4}, \mathrm{CO}_{2}$, and
$\mathrm{CO}$ are added. Since the shock tube involves the dissociation of air, $\mathrm{N}_{2}$, N and $\mathrm{NO}$ are also added. Thus, ten components are considered in the present study.

\subsubsection{Gas-phase friction and heat transfer terms}

The boundary layer along the tube wall is not modeled in the formulation of the gas dynamic equations. Instead, its effects are modeled by the addition of a wall shear in the momentum equation and heat transfer in the energy equation.

The wall-skin force on the gas is given by: $\tau=-\frac{1}{2} f \rho u|u| \pi \times$ $D \Delta x$. The skin friction coefficient for the pipe flow is then computed as [34]:

$$
\left\{\begin{array}{c}
f^{\prime}=0.049\left(\operatorname{Re}_{D}^{\prime}\right)^{-0.2}, \operatorname{Re}_{D}^{\prime} \geq 5507 \\
f^{\prime}=0.00875,5507 \geq \operatorname{Re}_{D}^{\prime} \geq 1828 \\
f^{\prime}=\frac{16}{\operatorname{Re}_{D}^{\prime}}, 1828 \geq \operatorname{Re}_{D}^{\prime}
\end{array}\right.
$$

$f=f^{\prime} \frac{T}{T^{\prime}}$ is evaluated at the reference temperature as follows: $T^{\prime}=0.9 T+0.03 M^{2} T+0.46 T_{w}$, where $T$ is the cell temperature and $T_{w}$ is the specified wall temperature. The Reynolds number is calculated as: $\operatorname{Re}_{D}^{\prime}=\frac{\rho^{\prime} u D}{\mu^{\prime}}$, where $u$ is the cell velocity and $D$ is the tube diameter of the cell. $\rho^{\prime}$ and $\mu^{\prime}$ are evaluated at the reference conditions: $\rho^{\prime}=\rho \frac{T}{T^{\prime}}, \mu^{\prime}=\mu^{\prime}\left(T^{\prime}, \rho^{\prime}\right)$.

The heat transfer from the gas to the walls is given by: $q=h \pi D \Delta x\left(T_{w}-T^{\prime}\right)$, where the heat transfer coefficient is $h=$ $\rho C_{p}|u| S t$ and the Stanton number is given by the modified Reynolds analogy for pipe flows [18]:

$S t=\frac{f}{8} \stackrel{-2 / 3}{\operatorname{Pr}}$

In the treatment of the viscous gas mixture, Wilke's mixturerule is applied with Maxwell's Power Law to calculate the dynamic viscosity of the gas [35]:

$\mu=\mu_{0}\left(\frac{T}{T_{\text {ref }}}\right)^{n}, n=0.76$ (air), 0.83 (detonation gas)

According to a previous report [18], the Prandtl number is given approximately as:

$\operatorname{Pr}=\frac{20 \gamma}{39 \gamma-15}$

\section{Results and discussion}

\subsection{Grid independence study}

A grid convergence study was conducted using the above viscous terms with three different grid resolutions $(3,421,6,841$ and $13,681)$. For simplicity, the dump section was not considered here. The simulations were performed for case 1 . It was found that the attenuation of the incident shock wave velocity obtained with three grid resolutions is basically is almost the same, as shown in Fig. 2. Additionally, there was also a negligible difference for the stagnation pressure for all grids, as shown in Fig. 3. Eventually, the grid with 6,841 grid points was used in the present study.

\subsection{Incident shock speed deceleration}

Characterization of the reservoir conditions for a shock tube/ tunnel is important, since it is generally used to estimate the free stream condition of the nozzle under a predictive method. To determine the reservoir conditions, the incident shock velocity and initial pressure/temperature values in the shock tube are needed. The initial pressure/temperature values in the shock tube are static 


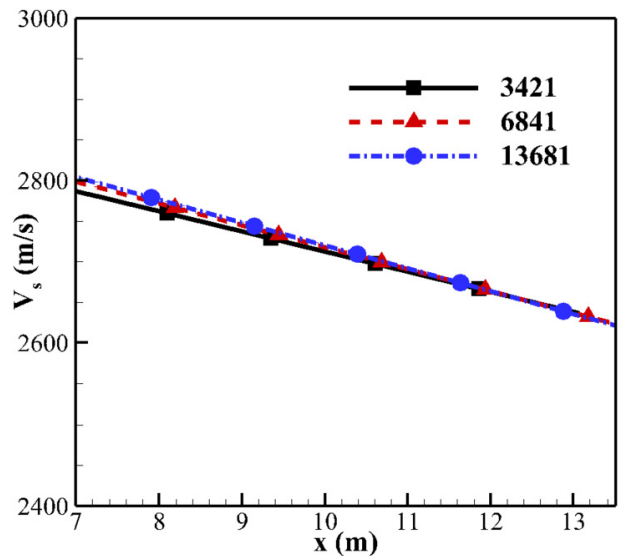

Fig. 2. Distribution of the incident shock wave velocity in the driven section for three grid resolutions.

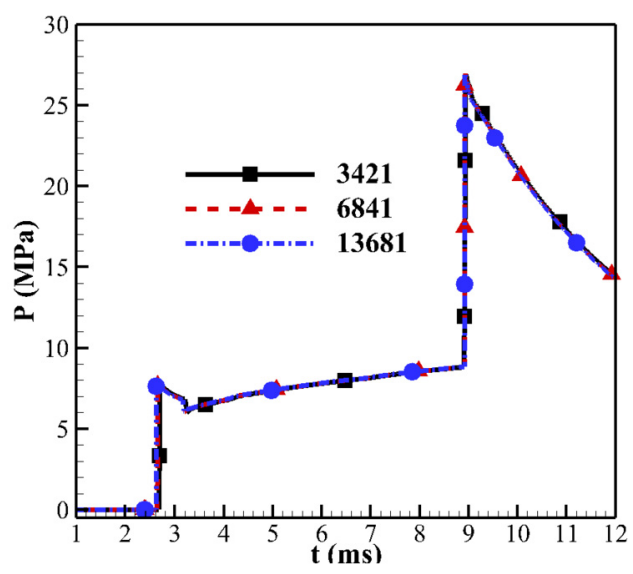

Fig. 3. Stagnation pressure histories at station P1 for three grid resolutions.

measurement and the uncertainty should be small. Thus, the incident shock speed here has significant influence to the reservoir parameters. The shock speed in the present study is measured at timing stations using ion probes, as mentioned above. Due to the viscous interaction and heat transfer between the shocked gas and the shock tube wall, the incident shock is attenuated. Other researchers have introduced an area change source term in the quasi-one-dimensional flow to study the effects of boundary layer growth on the attenuation of the shock speed. However, the boundary layer growth in the detonation tube was not defined. In this study, the pipe flow of skin friction correlations and modified Reynolds analogy for turbulent flow are introduced to estimate the shock attenuation.

The deceleration predicted by the model in section 2.2 is much lower than the experimental measured, as shown in Fig. 4. Such discrepancy is attributed to many factors, like the diaphragm opening effects, driver gas contaminations, shock/boundary layer interaction and tube wall roughness, which are difficult to model in a quasi-one-dimensional simulation. Thus, a scaling factor is used to better match the shock deceleration profile, as shown in Equation (6). Or rather, the scaling factor here does not merely represent the tube wall friction; it should be treated as the comprehensive result of all the factors. This factor may be different for different facilities and is suggested to be obtained by detailed comparison with experimental data. And as expected, a larger scaling factor results in higher shock deceleration since the wall-skin force on the gas is increased. A scaling factor of zero corresponds to the inviscid case with no wall-skin force on the gas, which corresponds to the Euler

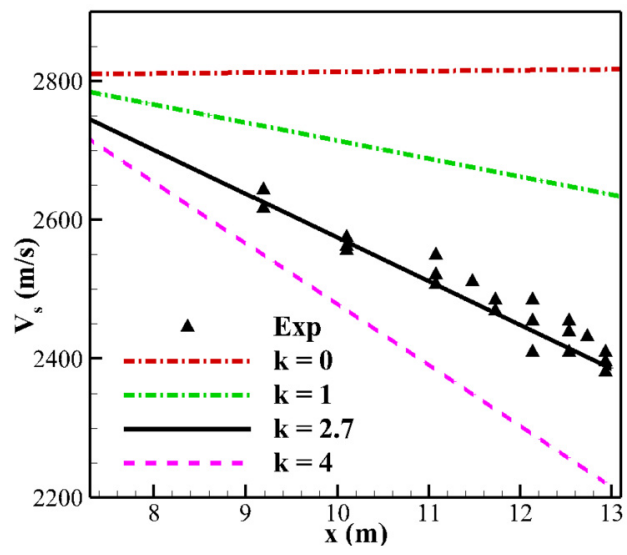

Fig. 4. Comparison of the incident shock velocity profiles. (For interpretation of the colors in the figure(s), the reader is referred to the web version of this article.)

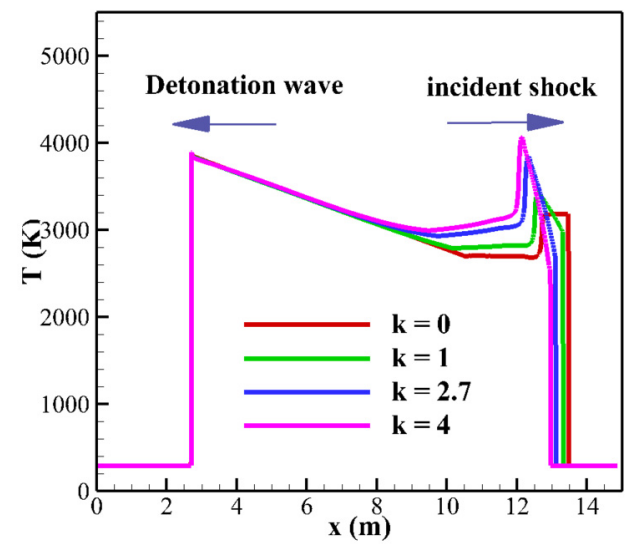

Fig. 5. Comparison of the temperature profiles in the shock tube at $\mathrm{t}=2 \mathrm{~ms}$.

simulation, and hence as expected the shock speed remains constant. It is revealed that a scaling factor of 2.7 corresponds better to the experimental decelerating case.

$\tau=k \times\left(-\frac{1}{2} f \rho u|u| \pi D \Delta x\right)$

The temperature distribution in the shock tube at $t=2 \mathrm{~ms}$ is shown in Fig. 5. It shows that different scaling factors cause the temperature difference of the driven section after the incident shock wave. As illustrated in Fig. 4, a larger scaling factor means that under the same conditions, more wall-skin forces work on the moving gas as shown the Fig. 6. Additionally, it can result in higher shock deceleration, which will lead to a lower temperature right after the incident shock, just as the temperature distribution in the shock tube in Fig. 5. However, it is interesting to find that the temperature of the test flow close to the interface in region 2 follows different laws. A larger scaling factor will result in a higher temperature for the test flow close to the interface. The calculation results of the inviscid and applied viscosity reveal that the effect of viscosity and heat transfer term considerably influence the temperature of the test gas during the process of wave motion. Accordingly, the selection of an appropriate numerical factor determines whether the calculation model is closer to the real physical process. The use of the experimental results of the incident shock wave velocity to properly modify the calculation method can ensure that the one-dimensional calculation method represents the influence of viscosity and heat transfer effects more accurately. Additionally, it can also be sees that although the viscosity significantly influences the shock deceleration in the shock tube, it has little effect on the detonation wave, where the detonation wave 


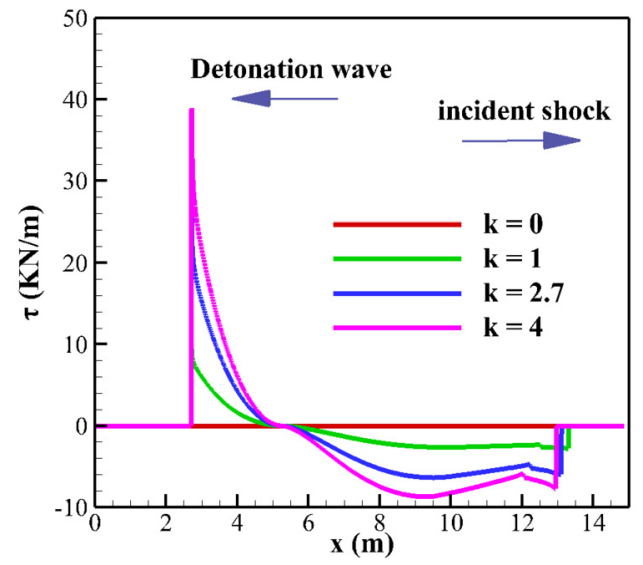

Fig. 6. Comparison of the wall-skin force per unit length profiles in the shock tube at $\mathrm{t}=2 \mathrm{~ms}$.

keeps the same speed for the four scaling factors. The reason for this will be analyzed in detail in the following content.

The temperature distributions, gas velocity distributions and wall-skin forces distributions in the shock tube for $\mathrm{k}=2.7$, at different time moments, are shown in Fig. 7. As time increases, the incident shock speed and the wall-skin forces decrease, the temperature right after the incident shock decreases, whereas the temperature close to the interface increases. Considering this problem from the point of view of energy conservation, the energy can be simply divided into three parts: the kinetic energy of the test flow, the internal energy of the test flow and the heat transfer from the gas to the wall. The kinetic energy and internal energy can be directly characterized by the speed and temperature of the test gas, while the heat transfer from the gas to the wall is determined by the work performed by the gas-phase friction. The effects of wall-skin forces are obvious at the initial moment due to the higher incident shock speed, and most of the kinetic energy loss is converted into the heat transfer from the gas to the wall. Since the incident shock speed decreases with the time extension, the test flow velocity after the shock will decrease. As a result, the effects of the wall-skin forces are reduced, and the change of the kinetic energy of the gas is converted into more internal energy of the gas itself than into the heat transfer to the wall. Thus, the increase of the internal energy of the test gas leads to an increased temperature, as shown in Fig. 7(a). The non-uniform distribution of the experimental gas state caused by the attenuation of the incident shock wave will affect the evaluation of the real experimental state. Even worse, the non-uniform temperature distribution would result in poor quality stagnation parameters after the shock reflection. In addition, it can lead to the conclusion that it is not reasonable to evaluate the reservoir state of shock tube by the conventional method based on the velocity of the shock wave without attenuation or the velocity of the shock wave decaying to the end of the shock tube.

For shock tunnels, the state parameters of region 5 are the most important. They determine the flight conditions that a shock tunnel can replicate. As mentioned above, when the incident shock wave is reflected at the shock tube end wall, it can produce higher temperature and pressure than in the region 2 . Thus, different incident shock states can produce different temperature and pressure states. The temperature history of region 5 with different scaling factors is shown in Fig. 8. It clearly reveals that the average temperature of the reservoir conditions without viscosity is nearly $1,000 \mathrm{~K}$ lower than that with viscosity $(\mathrm{k}=2.7)$. According to the change in temperature, the viscosity effect has a great influence on the state of the shock tunnel, which cannot be ignored in the process of numerical simulation. Besides, as shown in Fig. 8, the
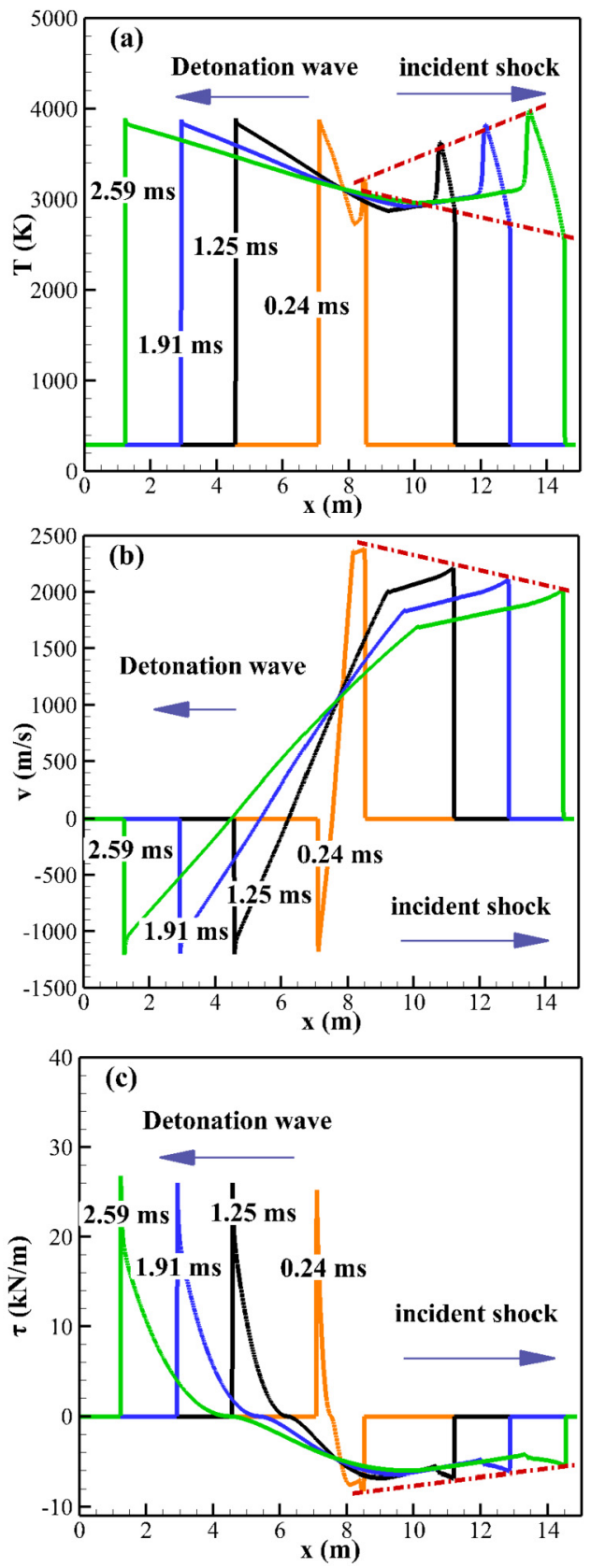

Fig. 7. Parameters distribution in the shock tube for $k=2.7$ (a) temperature, (b) gas velocity and (c) wall-skin forces per unit length.

reservoir conditions of temperature are not constant; instead, they are varying (increasing) over time. And unlike the stagnation pressure, this value is really hard to obtain experimentally under such a high temperature during several milliseconds. Thus, the present numerical method is also an effective tool for characterization of the reservoir temperature distribution in a shock tube/tunnel. In the following section, the pressure histories will also be compared with the experimental data.

\subsection{Detonation process}

Since the detonation wave propagation is a self-sustaining process, the dissipation effects have little influence on the performance of the detonation wave. This could be also obtained from the experimental results that the detonation wave velocity and $\mathrm{CJ}$ pressure do not decrease significantly as it propagates along the 


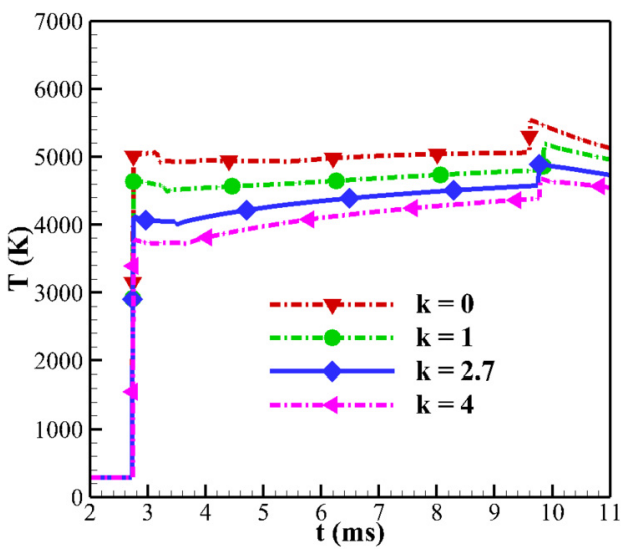

Fig. 8. Temperature history at station P1 with different scaling factors.

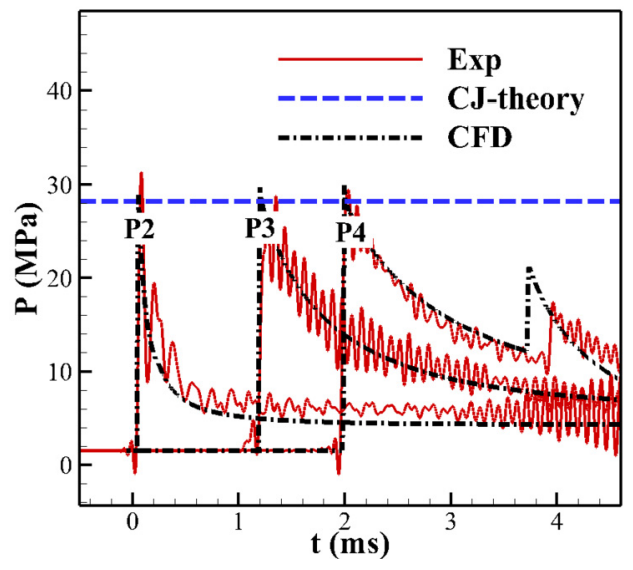

Fig. 9. Pressure histories measurements and calculated at stations $\mathrm{P} 2, \mathrm{P} 3$ and $\mathrm{P} 4$.

tube. Different scaling factors in the detonation tube have also been tested and they have little influence to the calculation results as shown in Fig. 5. Thus, the same scaling factor as in the shock tube part, that is $\mathrm{k}=2.7$, is adopted in the detonation tube for simplicity. The calculated pressure histories, recorded at stations P2, P3 and P4 in the driving section, and the experiments results, which were performed to validate the numerical simulation using the detonation driven shock tube, are shown in Fig. 9. It can be found that the arrival time lags of the simulation in the detonation wave between P2, P3 and P4 are almost the same as the experimental ones, which indicates an excellent agreement between the predicted and experimental detonation wave velocities. The calculated pressure histories also agree well with the observed ones, which show an increase after the arrival of the detonation wave and attenuation with the rarefaction wave. Simultaneously, the experimental and numerical value of the $\mathrm{CJ}$ pressure can match well with the Chapman-Jouguet theory. However, the estimated arrival time of the reflected detonation wave at station P4 is $\Delta t=1.7 \mathrm{~ms}$ after the detonation wave, which is a little shorter than the experimental value of $\Delta t=1.9 \mathrm{~ms}$. The reflection of detonation wave would also be affected by many factors, such as the diaphragm opening effects, shock/boundary layer interaction and tube wall roughness. Besides, there are not merely the auxiliary diaphragm exist between the driving and dump section; instead, complicated three-dimensional structures exist to weaken the high detonation pressure in reality. However, these factors are difficult to model in a quasi-one-dimensional simulation, even in a two-dimensional simulation. Considering that such attenuation has little impact on the results of the reservoir pressure, it is acceptable to deal with the detonation process by current numerical method.

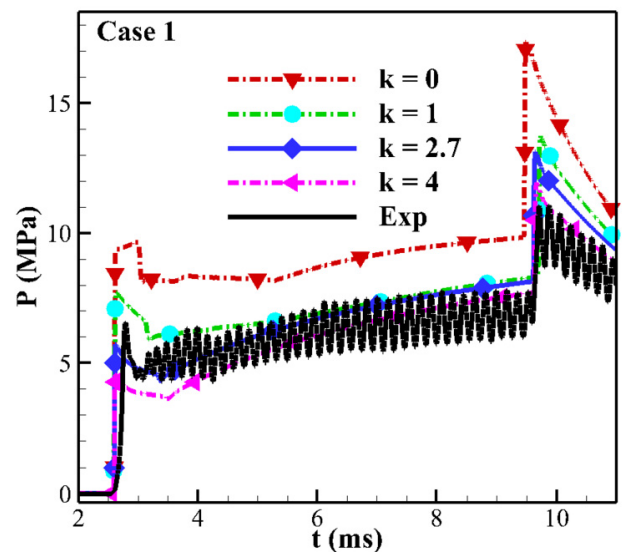

Fig. 10. Comparison of the reservoir pressure histories under case 1.

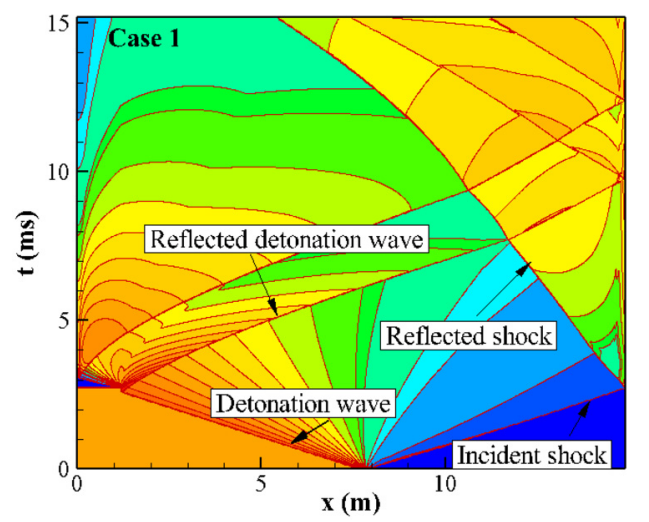

Fig. 11. $x$ - $t$ diagrams of the density contour for $\mathrm{k}=2.7$ under case 1 .

\subsection{Comparison of simulations to experimental results}

Calibration is performed by measuring parameters at various positions within the facility and then comparing the measurements with those of currently available modeling. The characterization of the flow at the inlet of the facility nozzle (reservoir conditions) is of critical importance. The pressure gauges at the end of the shock tube are used to record the time histories of the reservoir pressure. The magnitude of the reservoir pressure is mainly related to the intensity of the incident shock and the initial state parameters of the driven section. It was found that the simulated results agreed well with the experimental results for $\mathrm{k}=$ 2.7 compared with $\mathrm{k}=0, \mathrm{k}=1$ and $\mathrm{k}=4$ (see Fig. 10). The reservoir pressure of $\mathrm{k}=0$ and $\mathrm{k}=1$ were higher than the experimental ones due to their overestimated shock speed, while the stagnation pressure of $\mathrm{k}=4$ may be lower as result of the effects of the higher gas-phase friction. The results of various cases also show that the influence of the boundary-layer friction and heat transfer are important if accurate predictions of flow properties are expected.

To better understand the experimental results, the $x-t$ wave diagram for $\mathrm{k}=2.7$ is shown in Fig. 11 , which clearly illustrate the phenomenon of wave propagation. The increase of the pressure at $t=2.5 \mathrm{~ms}$ is due to the incident shock reflection, where the second increase at $t=9.3 \mathrm{~ms}$ is the arrival of the reflected detonation wave from the auxiliary diaphragm. The time interval between them can be used as reservoir condition for a shock tunnel.

For the other three test conditions, the numerical results are also compared with the experimental results. The distributions of the incident shock velocity and pressure history at the stagnation point are shown in Fig. 12 and Fig. 13, respectively. The total enthalpy values of the four conditions (including case 1) simu- 


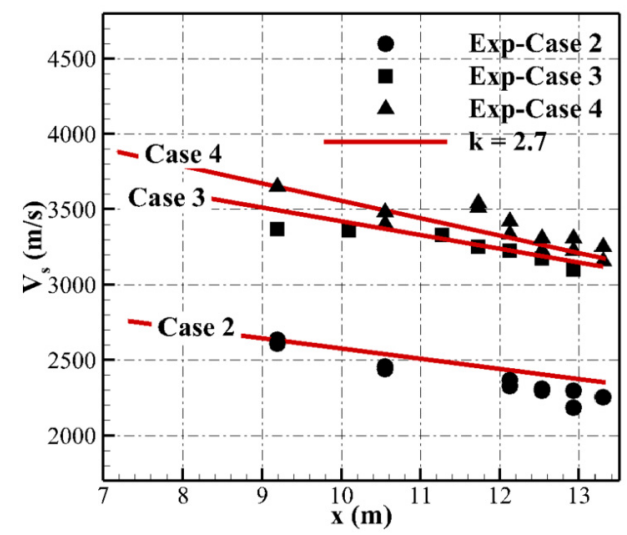

Fig. 12. Comparison of the distribution of the incident shock speeds in the shock tube.
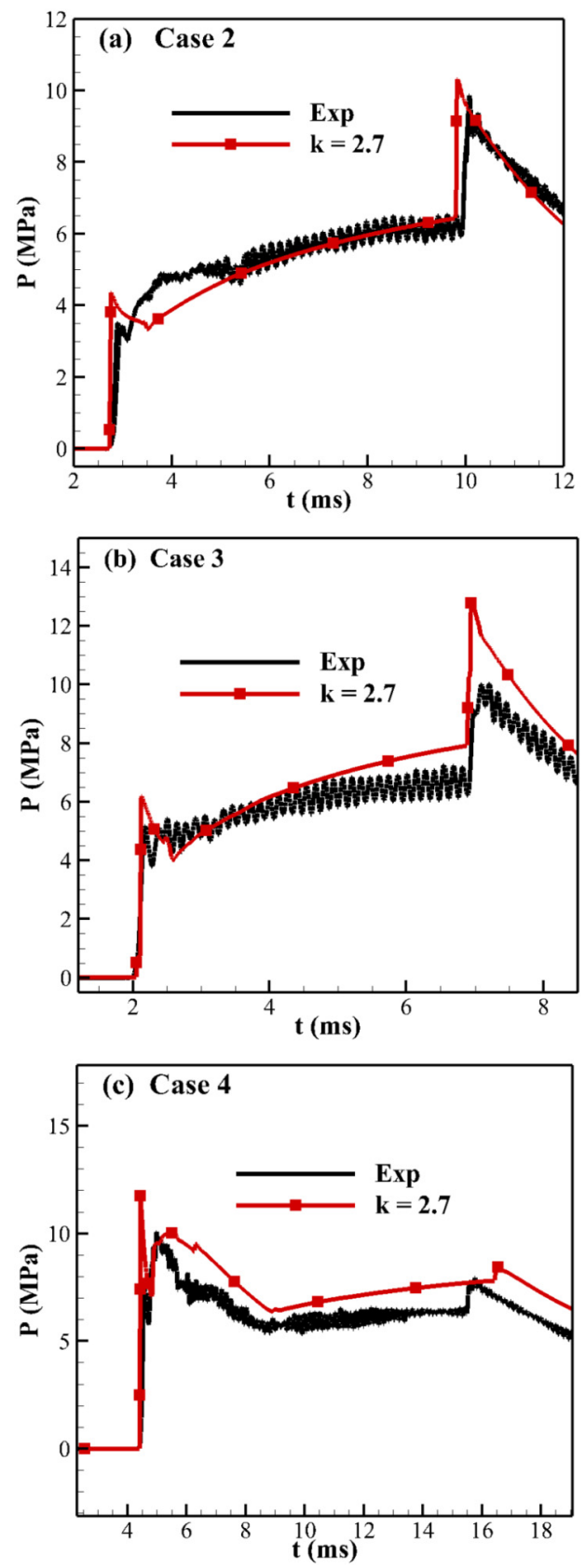

Fig. 13. Comparison of the reservoir pressure histories under three conditions. lated by the quasi-one-dimensional program covers from 6.8 to $13.5 \mathrm{MJ} / \mathrm{kg}$. The comparison revealed that the calculations of the quasi-one-dimensional program with $\mathrm{k}=2.7$ agrees well with the experimental results for cases 1 to 3 . However, there is little deviation for case 4 as shown in Fig. 13 (c). Case 4 is conducted under the forward detonation driving mode for a higher enthalpy value. However, the strong interaction between the detonation wave and the diaphragm cannot be modeled accurately. And the interface in this case is not precisely tailored. Thus, the pressure waves generated by the complicated interaction between the reflected shock and interface may return to the reservoir conditions. Nevertheless, the basic trend of the reservoir pressure is similar, and the deviation is acceptable in the present study.

In all, the present numerical method a simple tool for the design and assessment of new operating parameters of the test conditions in a detonation facility. Besides, it can also be used for characterization of the reservoir parameters in a shock tube/tunnel, especially for the total temperature which are difficult to measure in such facilities.

\section{Conclusions}

The aim of this study was to develop an accurate quick computation tool to simulate the flow in the detonation shock tube. It can explain the wave propagation process in the shock tube and provide relevant guidance for the rapid design of the shock tube operation state. The requirement of such a model arises because the different tests are run every day in the experimental facility, which needs to be simulated individually. Therefore, a quasi-onedimensional model is developed to simulate the shock tube flows.

The results under the different conditions tested here show that the quasi-one-dimensional modeling of detonation shock tunnels is an effective way to generate facility performance data. Although this paper has focused on the JFX shock tunnel, the modeling is generic and may be applied to other facilities.

\section{Declaration of competing interest}

The authors declare that there is no conflict of interests regarding the publication of this paper.

\section{Acknowledgements}

This work was supported by the National Natural Science Foundation of China (Grant Nos. 11402275, 11972331 and 11672308), which is gratefully acknowledged by the authors. The experimental support of the laboratory and fruitful scientific discussions with the members of the Laboratory are also gratefully acknowledged.

\section{References}

[1] H. Hornung, J. Belanger, Role and techniques of ground testing for simulation of flows up to orbital speed, in: 16th Aerodynamic Ground Testing Conference, 1990, p. 1377.

[2] S.D. Gu, H. Oliver, Capabilities and limitations of existing hypersonic facilities, Prog. Aerosp. Sci. 113 (2020) 100607, https://doi.org/10.1016/j.paerosci.2020. 100607.

[3] E.M. Dan, K.L. Frank, Advanced hypersonic test facilities, Prog. Astronaut. Aeronaut. (2002), https://doi.org/10.2514/4.866678.

[4] P. Sagnier, J.L. Vérant, On the validation of high enthalpy wind tunnel simulations, Aerosp. Sci. Technol. 2 (7) (1998) 425-437, https://doi.org/10.1016/ S1270-9638(99)80002-9.

[5] S.L. Gai, N.R. Mudford, Stagnation point heat flux in hypersonic high enthalpy flows, Shock Waves 2 (1992) 43-47, https://doi.org/10.1007/BF01414420.

[6] W. Zhao, Z.L. Jiang, et al., Performance of a detonation driven shock tunnel, Shock Waves 14 (2005) 53-59, https://doi.org/10.1007/s00193-004-0238-1.

[7] G.A. Bird, A note on combustion driven tubes, AGARD Report, 1957. 
[8] B.H.K. Lee, Detonation-driven shocks in a shock tube, AIAA J. 5 (1967) 791-792, https://doi.org/10.2514/3.4065.

[9] H.R. Yu, B. Esser, et al., Gaseous detonation driver for a shock tunnel, Shock Waves 2 (1992) 245-254, https://doi.org/10.1007/BF01414760.

[10] J.I. Erdos, R.J. Bakos, et al., Dual mode shock-expansion/reflected-shock tunnel, in: 35th Aerospace Sciences Meeting and Exhibit, 1997, p. 560.

[11] F.K. Lu, D.R. Wilson, Recent advances in detonation techniques for high enthalpy facilities, AIAA J. 38 (9) (2000) 1676-1684, https://doi.org/10.2514/2. 1153.

[12] Z.L. Jiang, H.R. Yu, Theories and technologies for duplicating hypersonic flight conditions for ground testing, Natl. Sci. Rev. 4 (2017) 290-296, https://doi.org/ 10.1093/nsr/nwx007.

[13] B.Q. Meng, G.L. Han, et al., Numerical investigation of the axial impulse load during the startup in the shock tunnel, Aerosp. Sci. Technol. 73 (2018) 332-342, https://doi.org/10.1016/j.ast.2017.12.013.

[14] D.V. Kotov, H.C. Yee, et al., Computational challenges for simulations related to the NASA electric arc shock tube (EAST) experiments, J. Comput. Phys. 269 (2014) 215-233, https://doi.org/10.1016/j.jcp.2014.03.021.

[15] A.M. Mohsen, M.Z. Yusoff, et al., Two-dimensional computational modeling of high-speed transient flow in gun tunnel, Shock Waves 28 (2018) 335, https:// doi.org/10.1007/s00193-017-0758-0.

[16] A.F. Amir, M.Z. Yusoff, et al., Numerical simulation of inviscid transient flows in shock tube and its validations, Int. J. Mech. Aerosp. Ind. Mechatron. Eng. 2 (7) (2010) 1-11.

[17] S. Chen, Q. Sun, I. Klioutchnikov, H. Olivier, Numerical study of chemically reacting flow in a shock tube using a high-order point-implicit scheme, Comput. Fluids 184 (30) (2019) 107-118, https://doi.org/10.1016/j.compfluid.2019. 02.019.

[18] P. Jacobs, Quasi-one-dimensional modeling of a free-piston shock tunnel, AIAA J. 32 (1) (1994) 137-145, https://doi.org/10.2514/3.11961.

[19] C. Mundt, R. Boyce, et al., Validation study of numerical simulations by comparison to measurements in piston-driven shock-tunnels, Aerosp. Sci. Technol. 11 (2-3) (2007) 100-109, https://doi.org/10.1016/j.ast.2006.12.002.

[20] K. Tani, K. Itoh, et al., Numerical study of free-piston shock tunnel performance, Shock Waves 3 (1994) 313, https://doi.org/10.1007/BF01415829.

[21] M.P. Sharma, One-dimensional modeling methodology for shock tubes: application to the EAST facility, in: 2018 Joint Thermophysics and Heat Transfer Conference, 2018, p. 4181
[22] Z.C. Owens, R.K. Hanson, The influence of wall heat transfer, friction, and condensation on detonation tube performance, Combust. Sci. Technol. 182 (8) (2010) 1104-1140, https://doi.org/10.1080/00102200903485202.

[23] M.I. Radulescu, R.K. Hanson, Effect of heat loss on pulse-detonation-engine flow fields and performance, J. Propuls. Power 21 (2) (2005) 274-285, https://doi. $\operatorname{org} / 10.2514 / 1.10286$.

[24] J.W. Li, Y.X. Tan, et al., Performance of a detonation driven shock tunnel, in: 21st AIAA International Space Planes and Hypersonics Technologies Conference, 2017, p. 2306.

[25] Z.L. Jiang, K. Takayama, An investigation into the validation of numerical solution of complex flow-fields, J. Comput. Phys. 151 (2) (1999) 479-497, https:// doi.org/10.1006/jcph.1999.6186.

[26] T. Ishihara, Y. Ogino, et al., Numerical study on wall pressure over cone region of blunt-nosed body in high enthalpy shock tunnel HIEST, Aerosp. Sci. Technol. 50 (2016) 256-265, https://doi.org/10.1016/j.ast.2015.12.015.

[27] J. Peng, Z.J. Zhang, et al., A theoretical and computational study of the vibration excitation on the transition criteria of shock wave reflections, Aerosp. Sci. Technol. 89 (2019) 299-306, https://doi.org/10.1016/j.ast.2019.03.021.

[28] H. Chen, C.Y. Wen, et al., Numerical simulation of air-He shock tube flow with equilibrium air model, AIAA J. 50 (9) (2012) 1817-1825, https://doi.org/10. 2514/1.J051129.

[29] C.P.T. Groth, J.J. Gottlieb, et al., Numerical investigation of high-temperature ef fects in the UTIAS-RPI hypersonic impulse tunnel, Can. J. Phys. 69 (7) (2011) 897-918, https://doi.org/10.1139/p91-144.

[30] W.R. Smith, R.W. Missen, Chemical Reaction Equilibrium Analysis: Theory and Algorithms, John Wiley, New York, 1982.

[31] B.J. McBride, M.J. Zehe, et al., NASA Glenn coefficients for calculating thermodynamic properties of individual species, NASA TP-2002-211556, 2002

[32] K. Hyungwon, K.L. Frank, et al., Numerical simulation of detonation process in a tube, Comput. Fluid Dyn. J. (2003).

[33] Y.S. Fang, Z.J. Zhang, et al., Initiation of oblique detonation waves induced by a blunt wedge in stoichiometric hydrogen-air mixtures, Aerosp. Sci. Technol. 92 (2019) 676-684, https://doi.org/10.1016/j.ast.2019.06.031.

[34] D.W. Bogdanoff, R.J. Miller, New Higher-Order Godunov Code for Modelling Performance of Two-Stage Light Gas Guns, 1995.

[35] R.S.M. Chue, C.Y. Tsai, et al., Driver gas contamination in a detonation-driven reflected-shock tunnel, Shock Waves 13 (2003) 367, https://doi.org/10.1007/ s00193-003-0221-2. 